\title{
ACESSO TRANSCONJUNTIVAL NA ABORDAGAGEM DE FRATURA DO ASSOALHO ORBITAL: RELATO DE CASO
}

\author{
TRANSCONJUNCTIVAL ACCESS IN ORBITAL \\ FLOOR FRACTURE: CASE REPORT
}

\begin{abstract}
Bruna Pedral Sampaio de Souza Dantas', Alana Del'Arco², Daniel Freitas', Roberto Almeida de Azevedo ${ }^{3}$, Laise Tourinho ${ }^{4}$

Autora para correspondência: Bruna Pedral Sampaio de Souza Dantas - brunapedral@yahoo.com.br 'Residentes de Cirurgia e Traumatologia Bucomaxilofacial da Universidade Federal da Bahia (UFBA), Salvador, Bahia, Brasil. ${ }^{2}$ Acadêmica do Curso de Odontologia da Universidade Federal da Bahia (UFBA), Salvador, Bahia, Brasil. ${ }^{3}$ Doutor em Odontologia. Professor de Cirurgia e Traumatologia Bucomaxilofacial da Faculdade na Universidade Federal da Bahia, Salvador, Bahia, Brasil.

${ }^{4}$ Cirurgiã Bucomaxilofacial, Salvador, Bahia, Brasil.
\end{abstract}

RESUMO | Objetivo: As fraturas na face necessitam de abordagens com resultados estéticos previsíveis, buscando acessos que minimizem cicatrizes e com baixos índices de complicações. Dentre os acessos extraorais para abordagem da margem infraorbital são descritos acessos cutâneos (subciliar, subtarsal e infraorbitário) e o transconjuntival com algumas variantes. Os acessos transconjuntivais possibilitam a exposição do assoalho da órbita, rebordo infraorbitário e parede medial da órbita para colocação de enxertos, osteotomias e ostessínteses, deixando cicatriz imperceptível na conjuntiva. O complexo zigomático está anatomicamente situado no terço médio da face e devido a sua projeção anteriorizada é bastante acometido por traumatismos, sendo a estrutura óssea facial, depois dos ossos próprios do nariz e da mandíbula, mais sujeita a fraturas. Descrição do caso: Este trabalho aborda caso clinico sobre o acesso transconjuntival para abordagem de fratura orbito-zigomatica de uma paciente gênero feminino JJC,30 anos, discutindo suas indicações, técnicas e complicações. Conclusão: A partir do diagnóstico, o tratamento proposto foi $\circ$ acesso transconjuntival para abordagem da fratura orbito-zigomática.

Palavras-chaves: fraturas zigomáticas, fraturas orbitárias, orbita

\begin{abstract}
Objective: Fractures in the face require approaches with predictable esthetic results, seeking access to minimize scars and to lower complication rates. Among the extraoral access to approach the infraorbital margin there are cutaneous accesses (subciliary, subtarsal and infraorbital) and Transconjunctival with some variations. The transconjuntivais access enable orbital floor display, infraorbital rim and medial orbital wall for placement of grafts, osteotomies and osteosynthesis, leaving imperceptible scars in the conjunctiva. The zygomatic complex is anatomically located in the midface and due to its anterior projection it is quite affected by trauma, and facial bone structure, after the nasal bones, more prone to fractures. Case Description: This paper discusses a clinical case on the transconjunctival access to orbitozygomatic fracture approach of a female patient JJC, 30, discussing their indications, techniques and complications. Conclusion: From the diagnosis, the recommended treatment was the transconjunctival access to approach orbitozygomatic fracture.
\end{abstract}

Keywords: zygomatic fractures, orbital fractures, orbit 


\section{INTRODUÇÃO}

As fraturas na face necessitam de abordagens com resultados estéticos previsíveis, buscando acessos que minimizem cicatrizes e com baixos índices de complicações. Dentre os acessos extraorais para abordagem da margem infraorbital são descritos acessos cutâneos (subciliar, subtarsal e infraorbitário) e o transconjuntival com algumas variantes'. A abordagem transcaruncular, utilizada no acesso à parede medial da órbita, pode estender-se e expandir superiormente, por trás do sistema de drenagem lacrimal ${ }^{2}$.

O primeiro relato do acesso transconjuntival foi feito por Bourguet em 1924 na blefaroplastia para remoção de gordura em pálpebra inferior ${ }^{3}$. Os acessos transconjuntivais possibilitam a exposição do assoalho da órbita, rebordo infraorbitário e parede medial da órbita para colocação de enxertos, osteotomias e ostessínteses, deixando cicatriz imperceptível na conjuntiva ${ }^{2}$. A dissecção da pele ou do músculo não é necessária sendo considerada uma abordagem de baixo tempo cirúrgico ${ }^{4}$.

Observa-se limitações quanto ao acesso dos ossos zigomático e maxilar, sendo assim, em algumas situações que maior exposição da área cirúrgica é necessária pode-se realizar a cantotomia. $O$ acesso transconjuntival com cantotomia lateral possibilita ao cirurgião adequada exposição do rebordo infraorbitário, margem lateral da órbita e assoalho orbitário².

Dentre as principais complicações desse acesso têm-se $\circ$ entrópio da pálpebra inferior, lesões diretas ao globo ocular, exposição de gordura orbital, ectrópio da pálpebra inferior, aumento na exposição da esclera, laceração da pele, injuria do sistema lacrimal, laceração da lâmina tarsal, mau posicionamento do ligamento lateral, granuloma conjuntival e cisto de inclusão ${ }^{5}$.

O complexo zigomático está anatomicamente situado no terço médio da face e devido a sua projeção anteriorizada é bastante acometido por traumatismos, sendo a estrutura óssea facial, depois dos ossos próprios do nariz, mais sujeita a fraturas. Apresenta quatro processos, o temporal, o orbital,
- maxilar e o processo frontal, nos quais compõem os pontos de fragilidade e maior índice de fratura ${ }^{6}$.

Este trabalho tem portanto o objetivo de apresentar um caso clinico sobre o acesso transconjuntival para abordagem de fratura orbito-zigomatica, discutindo suas indicações, técnicas e complicações.

\section{RELATO DE CASO (CAAE 60333416.8.0000.5024)}

Paciente JJC, gênero feminino, 30 anos, ASA I, natural de Jacobina - Bahia foi vítima de acidente motociclístico no dia 05/10/14 e procurou o serviço de Cirurgia e Traumatologia Bucomaxilofacial do Hospital Santo Antônio- UFBA para reabilitação.

A paciente apresentava como queixa principal "meu olho afundou". Na história médica pregressa negou patologias de base, assim como afirmava não possuir alergia medicamentosa, e não fazer uso crônico de medicamentos.

Durante a anamnese, paciente negou uso de drogas ilícitas, internamento prévio e transfusão sanguínea. Quanto ao histórico familiar paciente possuía pais hipertensos e pai diabético.

No exame físico da face observou-se acuidade visual e motilidade ocular preservada bilateral, equimose periorbital em olho direito em estágio de regressão, degrau ósseo em rebordo infraorbitário direito, hipoftalmo e enoftalmo em olho direito, hipoestesia em região infraorbitária à direita, ausência de mobilidade atípica a manipulação dos maxilares, boa abertura bucal e oclusão estável. (Figura 01 e 02) 


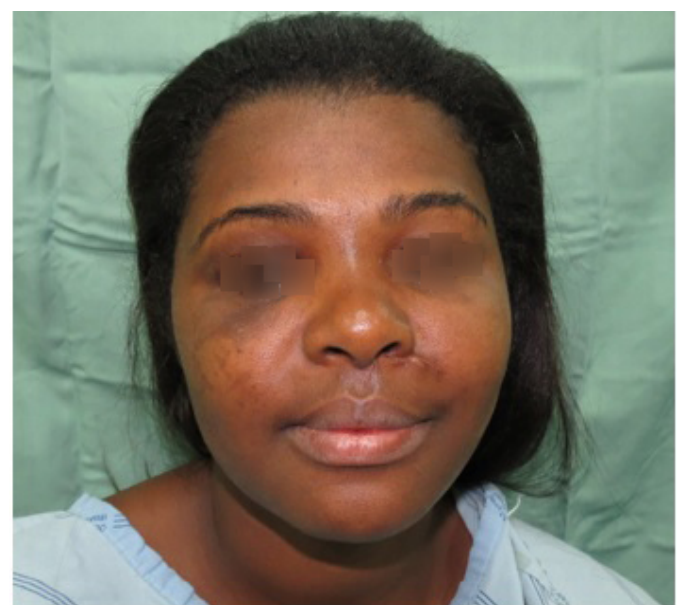

Figura 01. Foto frontal pré operatória pode ser observado o hipoftalmo

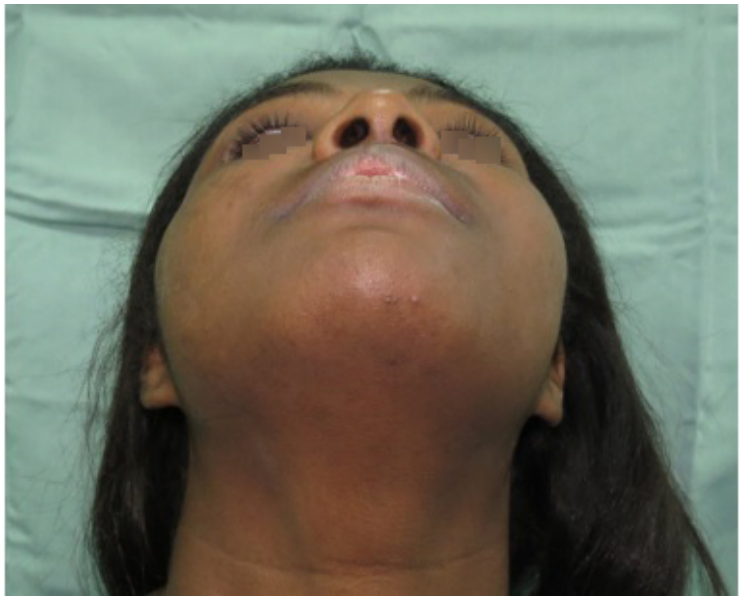

Figura 02. Foto mento naso pré operatória observa-se o enoftalmo

No exame complementar por imagem pré-cirúrgico nota-se na tomografia computadorizada sinais sugestivos de fratura do complexo zigomático direito. (Figuras 03-07).

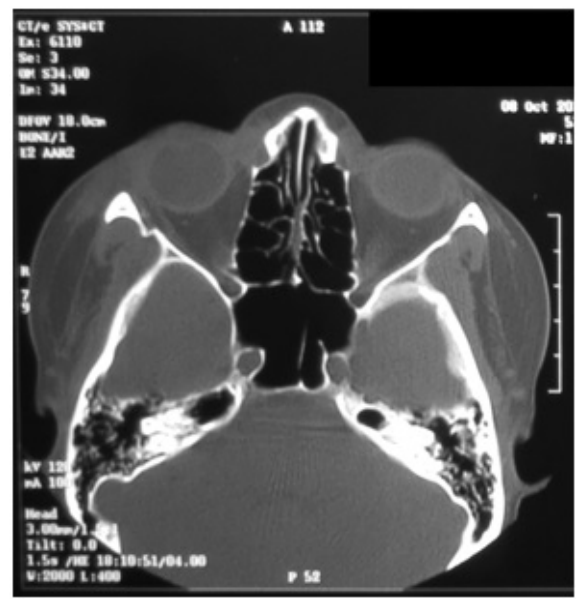

Figura 03. Corte axial (fratura parede lateral orbita direita)

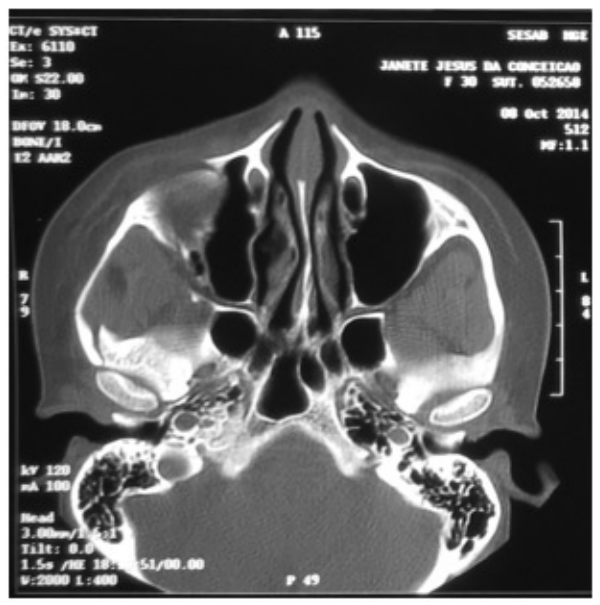

Figura 04. Corte axial

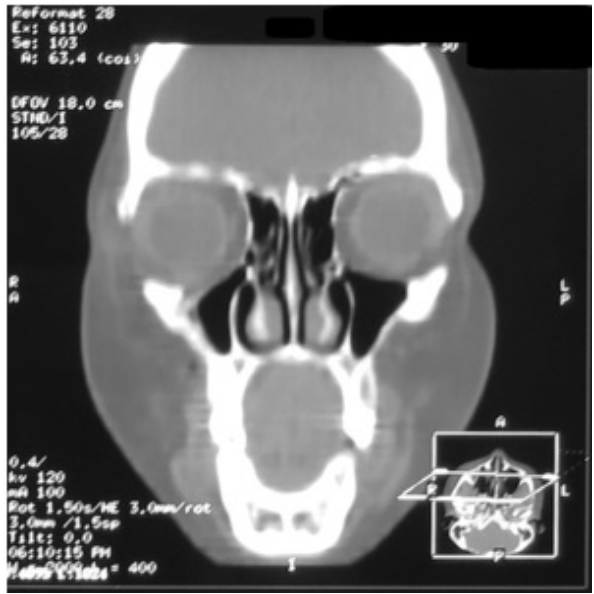

Figura 05. Corte coronal (fratura em rebordo infraorbitario direito)

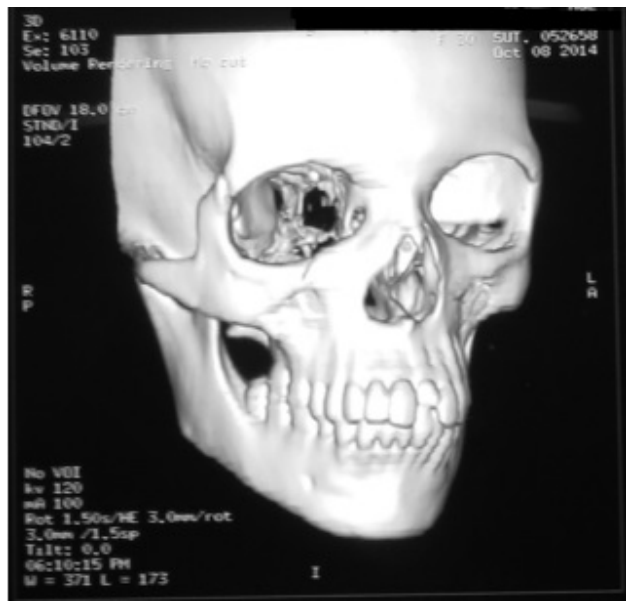

Figura 06. Reconstrução 3D (fratura em sutura frontomalar direita e rebordo infraorbitario direito) 


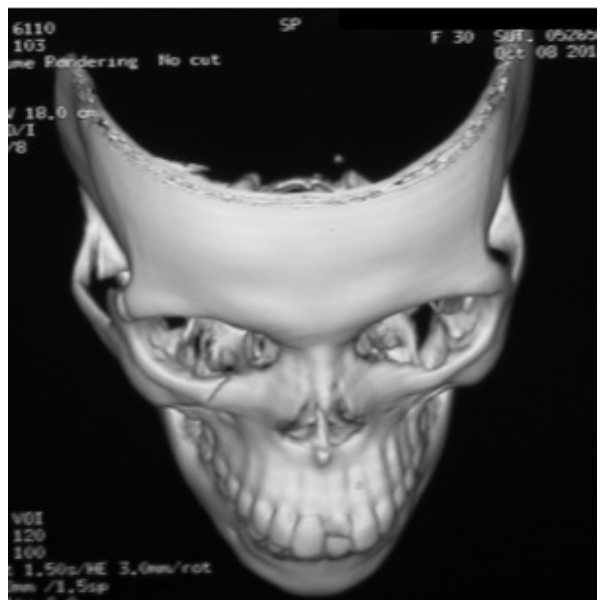

Figura 07. Reconstrução 3D (observa-se fratura em rebordo infraorbitario direito)

Diante da análise clínica e imaginológica a paciente foi submetida a cirurgia sob anestesia geral no dia 09/12/14 no Hospital Santo Antonio das Obras Sociais Irma Dulce. Realizou-se a inserção de lente de proteção ao globo ocular, acesso tipo transconjuntival com cantotomia lateral, exposição do traço de fratura, instalação de tela orbital em assoalho orbital e fixação com 01 parafuso do sistema $1.5 \mathrm{~mm}$ para correção do hipoftalmo e enoftalmo, fixação com 01 placa orbital do sistema $2.0 \mathrm{~mm}$ com 05 furos e 04 parafusos em região infraorbitária a direita e 01 placa do sistema $2.0 \mathrm{~mm}$ com 06 furos e 04 parafusos em região fronto malar a direita (Figura 08 e 09). Seguiu-se com irrigação abundante com soro fisiológico 0,9\% e sutura das feridas cirúrgicas com vicryl 4-0 e nylon 6-0 em região fronto malar.

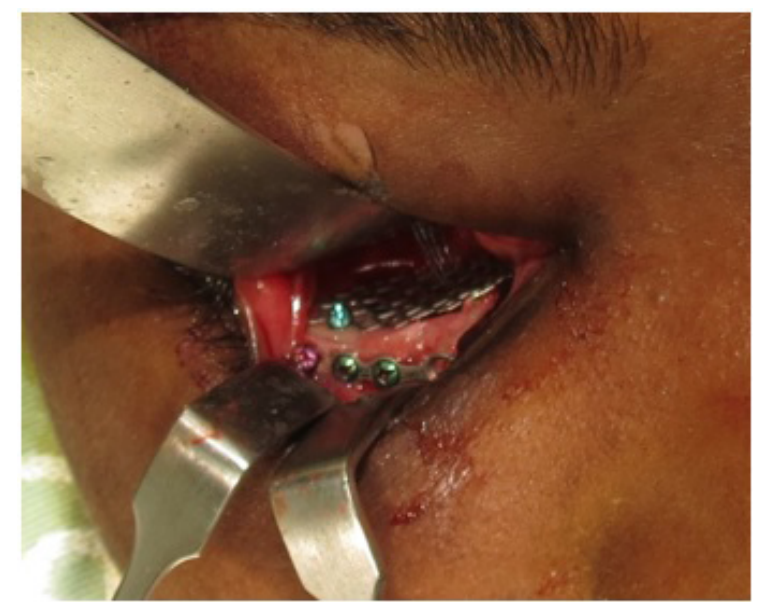

Figura 08. Acesso tranconsjuntival

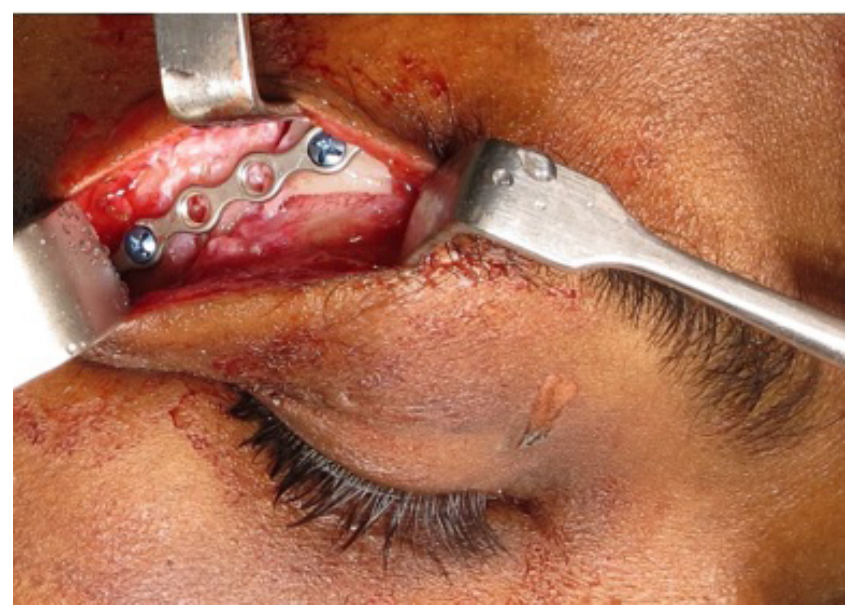

Figura 09. Acesso fronto malar

No exame por imagem pós-operatório de 01 ano da radiografia mentonaso, PA de face e telerrediografia de perfil verifica-se material de síntese em posição adequada, cicatrização óssea dentro da normalidade e aspecto final de acordo com o planejamento inicial (Figuras 10, 11 e 12).

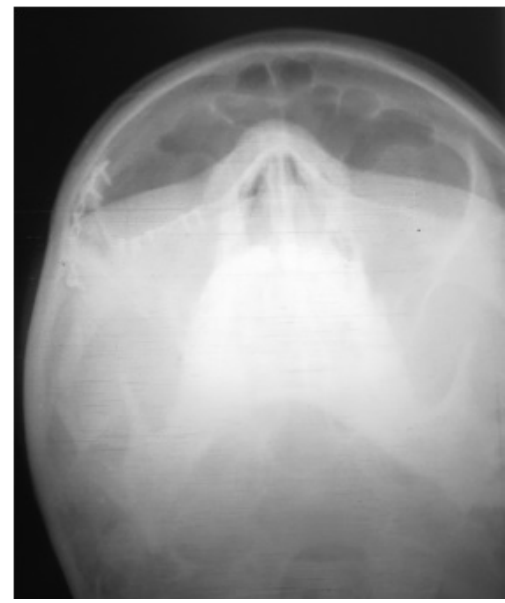

Figura 10. Rx Mentonaso

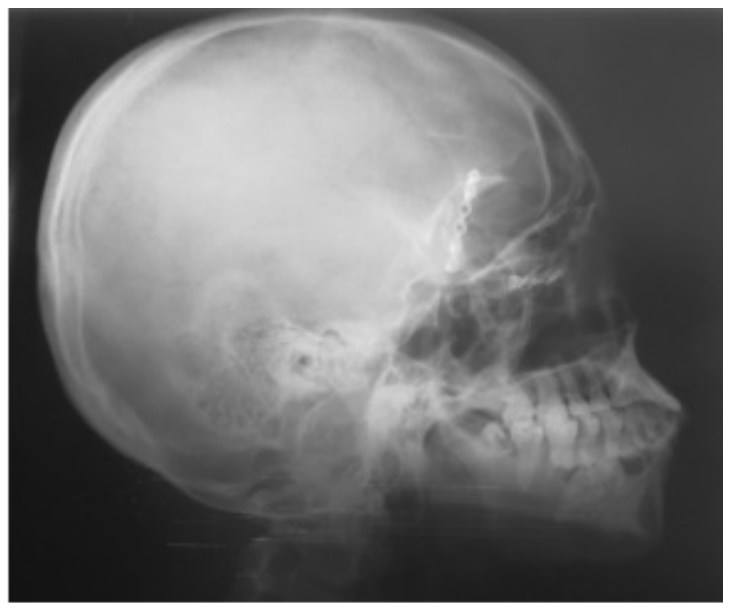

Figura 11. Telerradiografia de Perfil 


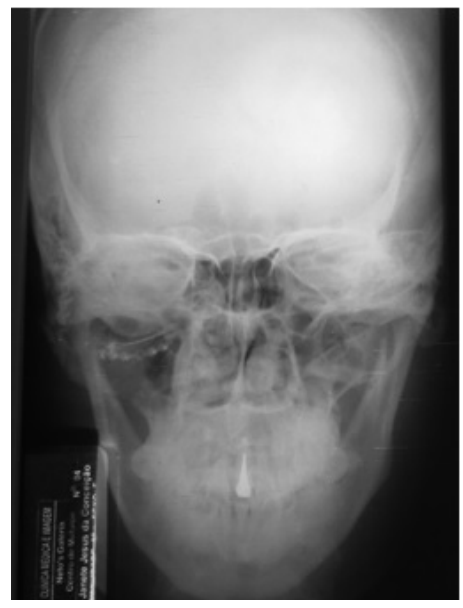

Figura 12. Rx PA de Face

Após 01 ano observou-se resultado satisfatório com cicatriz imperceptivel, sem sinais de complicações e discreta melhora do enoftalmo e hipoftalmo. (Figura 13 e 14)

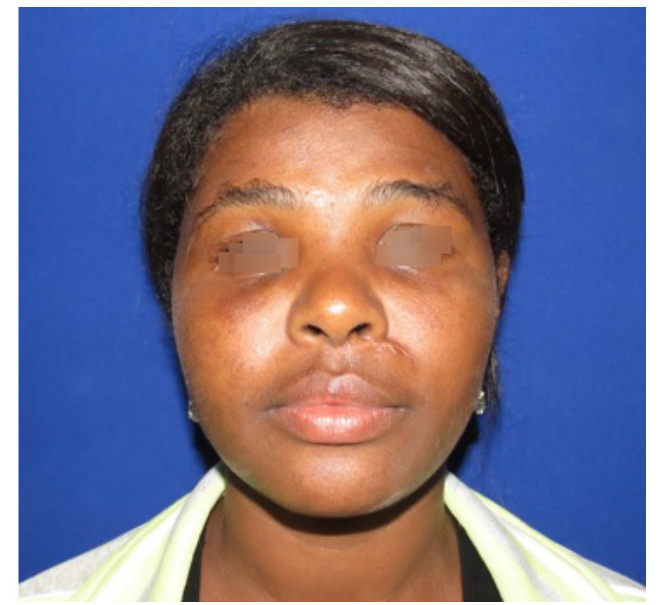

Figura 13. Foto frontal pós operatorio

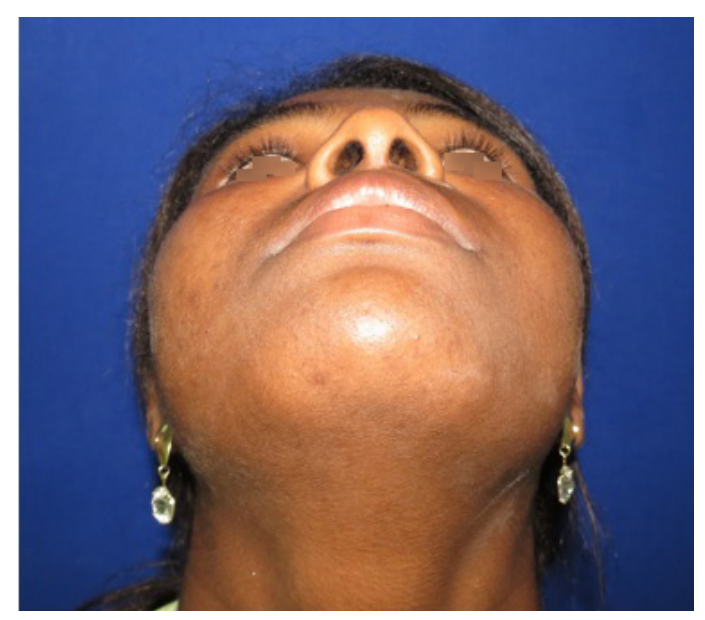

Figura 14. Foto mentonaso pós operatório

\section{DISCUSSÃO}

A escolha do acesso e do local da incisão pode ser orientada pelos seguintes objetivos: boa visualização intraoperatória, formação mínima de cicatriz pós-operatória e bom resultado estético². O seguinte relado de caso, a escolha pelo acesso transconjuntival com cantotomia lateral corrobora com esses requisitos, promovendo um excelente acesso e sem complicações no pós operatório.

Entre os diferentes tipos de acessos cirúrgicos para fratura de complexo zigomático, podemos citar - acesso subciliar, o qual promove um excelente acesso para a borda infra-orbital, parede lateral da órbita e todo o assoalho orbital, deixando uma cicatriz o qual fica recoberta pela sombra dos cílios, tornando-se imperceptível. Dentre suas vantagens podemos citar o amplo acesso ao assoalho orbital e borda infra-orbital, além da facilidade de extensão lateral, permitindo acesso a toda borda lateral da órbita, o fácil aprendizado. No entanto, exposição acentuada da esclera, ectrópio da pálpebra inferior, entrópio, exposição de córnea, abrasão da córnea, queimadura da córnea, lagoftalmo, astigmatismo, estrabismo, diplopia, assimetria entre os lados, ptose, conexão do canto medial, olho seco, retração são considerados algumas das complicações iatrogênicas dessa técnica ${ }^{7}$.

A abordagem subtarsal fornece adequada exposição cirúrgica da borda infraorbital e do assoalho da órbita, permitindo ampla e direta visualização, além de espaço suficiente para manuseio, disposição e adaptação do material de síntese ou de reconstrução no transoperatório. Como as complicações podemos citar: conjuntivite, epífora e cicatriz aparente. Se comparada com a técnica transconjuntival, os índices de ectrópio e exposição de esclera apresentados nesta abordagem são equivalentes ${ }^{5}$.

No acesso infraorbital uma incisão é realizada através da pele, tecido subcutâneo, músculo orbicular e periósteo, diretamente sobre a borda infraorbital, permanecendo intacto $\circ$ septo ${ }^{7}$. A incisão precisa afilar-se lateralmente, evitando lesão do sistema de drenagem linfática da pálpebra inferior, com consequente edema palpebral prolongado e comprometimento da cicatriz ${ }^{5}$. 
A cicatriz imperceptível, escondida na conjuntiva, técnica simples e os resultados funcionais e estéticos satisfatórios, é a principal vantagem da técnica transconjuntival ${ }^{4}$, o índice de complicação é baixo ${ }^{5}$ e, com uma baixa incidência de ectrópio ${ }^{5}$. Nota-se maior índice de complicações na técnica subciliar quando comparada à transconjuntival. $O$ acesso transconjuntival evita em maior eficiência o mau posicionamento cantal medial, telecanto atraumático e injúrias ao saco lacrimal em relação às técnicas transcutâneas. $8 \mathrm{Em}$ contrapartida, a incidência de epífora foi maior no acesso transconjuntival do que no acesso subciliar. (MORAIS 2013) A desvantagem comumente citada é a limitação de campo operatório, que pode ser contornado pela cantotomia lateral. Raras complicações temporárias têm sido citadas, como, por exemplo, a oftalmoplegia ${ }^{5}$.

No acesso transconjuntival, existem duas vias de dissecção que permitem o acesso cirúrgico, a via pré-septal e a via retrosseptal ${ }^{5}$. Na primeira, a dissecção caminha a frente do septo que isola o conteúdo adiposo da órbita, evitando o herniamento deste conteúdo para o sítio cirúrgico ${ }^{5,6}$. A incisão é feita através da conjuntiva, abaixo do tarso, e o plano de dissecação se localiza entre o músculo orbicular e o septo orbitário. $O$ fato de evitar a exposição do conteúdo adiposo da órbita facilita a visualização do campo operatório e, consequentemente, a redução e fixação adequada da fratura ${ }^{3,6}$. Já na via retrosseptal, a dissecção ocorre por via adiposa até o plano do periósteo, sendo necessário o cuidadoso afastamento deste tecido para visualização ideal do campo operatório. Em seguida, o periósteo é incisado e descolado para visualização dos cotos fraturados de forma direta $^{5}$. A herniação da gordura periorbitária pode resultar em reações indesejáveis como atrofia pósoperatória e retração palpebral ${ }^{6,7}$. A via pré-septal produz uma mínima cicatriz lateral, uma excelente aceitação pelo paciente, e diminui as chances de retração palpebral ou ectrópio?.

\section{DISCUSSÃO}

O acesso transconjuntival com cantotomia lateral possibilita ao cirurgião adequada exposição do rebordo infraorbitário, margem lateral da órbita e assoalho orbitário, com baixo índice de complicações e excelentes resultados estéticos. Desde que haja treinamento adequado do cirurgião, essa técnica oferece bons resultados estéticos e funcionais, minimizando cicatrizes faciais além de evitar incisões transcutâneas e suas possíveis complicações. Dessa forma, diante de um planejamento cirúrgico adequado analisando as particularidades de cada caso, o acesso transconjuntival deve ser realizado na busca de um resultado estético e funcional satisfatório.

\section{CONFLITOS DE INTERESSES}

Nenhum conflito financeiro, legal ou político envolvendo terceiros (governo, empresas e fundações privadas, etc.) foi declarado para nenhum aspecto do trabalho submetido (incluindo mas não limitandose a subvenções e financiamentos, conselho consultivo, desenho de estudo, preparação de manuscrito, análise estatística, etc).

\section{REFERÊNCIAS}

1. Ribeiro Neto N, Macedo Sobrinho JB, Pozza DH, Oliveira MA, Ribeiro MAN, Oliveira MG. Fraturas do arco zigomático: cerclagem de contenção. Clin. Pesq. Odontol. 2006;2(3):225229

2. Araúio MM, Cavalieri I, Pereira CCS, Oliva MA, Costa DA. Acesso Transconjuntival para fraturas do complexo zigomático-orbitário: Relato de caso. Rev. Cir. Traumatol. Buco-Maxilo-Fac. 2006;6(4):39-48

3. Morais HHA, Grempell RG, Barbalho JC, Sousa TG, Silva AL. Fratura blow out tratada com acesso transconjuntival e cantotomia lateral: relato de caso. Rev. Cir. Traumatol. BucoMaxilo-Fac. 2014;14(1):39-42

4. Novelli G, Ferrari L, Sozzi D, Mazzoleni F, Bozzetti A. Transconjunctival approach in orbital traumatology: A review of 56 cases. Journal of Cranio-Maxillo-Facial Surgery. 2011 ; 39(4):266-70. doi: 10.1016/i.jacms.2010.06.003

5. Ferreira FM, Zorzetto DLG, Marzola C, Toledo Filho JL, Pastori CM, Capelari MM, et al. Acessos cirúrgicos infraorbitais Revista da Literatura e relato de caso clínicocirúrgico. Bauru-SP

6. Angelim DD, Silva LF, Gondim RF, Bezerra Junior GL, Mello MJR, Carvalho ACGS. Acesso transconjuntival no tratamento de fraturas do osso zigomático - relato de caso. Rev. Odontologia (ATO). 2015;15(8):445-455 
7. Timóteo CA, Chagas JFS, Rapoport A, Denardin OVP. Avaliação da abordagem palpebral subtarsal no tratamento cirúrgico das fraturas zigomático-orbitais. Rev. Col. Bras. Cir. 2009;36(5):382-391. doi: 10.1590/S010069912009000500005

8. Suga H, Sugawara Y, Uda H, Kobayashi N. The transconjunctival approach orbital bony surgery: in which cases should it be used? The Journal of Craniofacial Surgery. 2004; 15(3): 454-457

9. Santosh BS, Giraddi G. Transconjunctival preseptal approach for orbital floor. J. Maxillofac. Oral Surg. $2011 ; 10(4): 301-305$. doi: 10.1007/s12663-011-0246-5 